\title{
Self-Consistency Validation of Subgrid Scale Parameterization Schemes in a Large-Eddy Simulation
}

\author{
Yuji KITAMURA \\ Meteorological Research Institute, Japan Meteorological Agency, Tsukuba, Japan
}

(Manuscript received 16 February 2010, in final form 21 June 2010)

\begin{abstract}
In the present study, we perform a set of numerical simulations in a moderately stable boundary layer with four types of subgrid-scale parameterization schemes and attempt to evaluate the error of the vertical flux for these schemes in terms of self-consistency on the basis of the Germano identity. If the effects of grid-scale components in higher wavenumbers are excluded from the analysis, the error estimated by the Germano identity is insensitive to the reference data utilized. The subgrid-scale flux, evaluated by the Smagorinsky model, tends to excessively weaken the positive temperature gradient at the top of the boundary layer with decreasing model resolution. The Deardorff and two-part models overestimate the subgrid-scale temperature flux at a coarser resolution, and the dynamic Smagorinsky model tends to underestimate both the subgrid-scale momentum and the temperature fluxes throughout the entire boundary layer. The underestimation of the subgrid-scale flux found in the dynamic Smagorinsky model could be attributed to a low correlation between the resolved and the parameterized components.
\end{abstract}

\section{Introduction}

Large-eddy simulation (LES) is a type of numerical approach that deals with equations describing fluid motion. In this approach, a physical quantity is divided into grid-scale (GS) and subgrid-scale (SGS) components with a spatial filtering operation (Leonard 1974). In an LES, the equations for GS motion with spatial scales larger than the grid spacing of a numerical model are explicitly computed and the contribution of an SGS component to GS motion is parameterized using additional assumptions, while only ensemble-averaged motions are solved in the Reynolds-averaged numerical simulation (RANS). A parameterization scheme that represents the effect of SGS motions was first proposed by Smagorinsky (1963), and schemes for SGS modeling have since been developed by many other re-

Corresponding author: Yuji Kitamura, Meteorological Research Institute, Japan Meteorological Agency, 1-1, Nagamine, Tsukuba, Japan.

E-mail: kitamura@mri-jma.go.jp

(C) 2010, Meteorological Society of Japan searchers. LES can be a powerful approach for numerical studies of fluid mechanics when the model resolution is sufficient to represent most turbulent eddies.

Deardorff (1972) first applied LES to the numerical simulation of a planetary boundary layer. Because of recent advancements in computational power, this method has been widely applied as a useful tool for performing numerical simulations of atmospheric boundary layers. However, numerical modeling in a stable boundary layer $(\mathrm{SBL})$ is still a difficult task because turbulent motion is vertically suppressed because of density stratification, and the characteristic scale of eddies becomes smaller. Hence, finer model resolution is required to resolve such eddies in the SBL. While the appropriateness of SGS modeling is important for reproducing realistic turbulent flows, a more appropriate way to represent SGS motions in the SBL has not been resolved at present.

An intercomparison of LES models for an SBL was conducted as part of the Global Energy and Water Cycle Experiment Atmospheric Boundary 
Layer Study (GABLS) initiative (Beare et al. 2006). Their results indicate that most SGS models are capable of reproducing the characteristic vertical structure of mean velocity and temperature (e.g., a nocturnal jet and a positive curvature in the temperature near the top of the SBL) with reasonable accuracy in a moderately stable stratification. However, distinct differences in SBL height and surfaceflux magnitude have also been observed among SGS models, even at 2-m resolution. It appears that it is necessary to quantitatively evaluate the accuracy of results obtained from each model. Beare et al. (2006) attempted to compare model results with the observation made by Nieuwstadt (1984) in order to investigate LES reliability. While the normalized momentum and heat fluxes obtained from the LES were consistent with observations, the presence of some errors in observational data (due to the difficulty of obtaining accurate measurements in the real atmospheric boundary layer) could prevent accurate comparisons between the model and observations. Such a situation motivates the search for a different method to validate the results of numerical simulations. It should also be noted that each model used in the intercomparison by Beare et al. (2006) adopts a different scheme of dynamics (e.g., an advection scheme) in the numerical simulation. An experiment performed with a common model would be necessary to extract the dependence on a subgrid scheme.

In the present study, we perform a set of numerical simulations in a moderately stable boundary layer following the GABLS intercomparison case with four types of subgrid-scale parameterization schemes (Smagorinsky, dynamic Smagorinsky, Deardorff, and two-part models). We attempt to examine the self-consistency of these schemes in terms of the Germano identity in order to investigate the appropriateness of the parameterized momentum and heat fluxes. While the Germano identity was originally used to determine the model constant that appeared in the dynamic Smagorinsky model so that the square error of the subgrid stress is minimized (Germano et al. 1991; Lilly 1992), Meneveau and Katz (1999) suggested that the Germano identity is also able to explicitly evaluate errors at scales between the grid and test filters. On the basis of this idea, we aim to evaluate the error in the vertical flux with data obtained from numerical models.

This paper is organized as follows. In Section 2, the numerical model and SGS schemes used in the present study are described. The results of an intercomparison of the mean profile and flux are presented in Section 3. Section 4 is devoted to selfconsistency validation of the LES models in terms of the Germano identity, which is the main part of this study. A summary is given in Section 5.

\section{Model description}

The governing equations of the grid-scale motion and temperature are described as follows:

$$
\begin{aligned}
& \frac{d \bar{u}}{d t}=-\frac{\partial \bar{p}}{\partial x}+f\left(\bar{v}-V_{g}\right)-\frac{\partial \tau_{1 j}}{\partial x_{j}}, \\
& \frac{d \bar{v}}{d t}=-\frac{\partial \bar{p}}{\partial y}-f\left(\bar{u}-U_{g}\right)-\frac{\partial \tau_{2 j}}{\partial x_{j}}, \\
& \frac{d \bar{w}}{d t}=-\frac{\partial \bar{p}}{\partial z}+\frac{g}{\theta_{0}}(\bar{\theta}-\langle\theta\rangle)-\frac{\partial \tau_{3 j}}{\partial x_{j}}, \\
& \frac{d \bar{\theta}}{d t}=-\frac{\partial \tau_{\theta j}}{\partial x_{j}} \\
& \frac{\partial \bar{u}}{\partial x}+\frac{\partial \bar{v}}{\partial y}+\frac{\partial \bar{w}}{\partial z}=0
\end{aligned}
$$

in an incompressible fluid on an f-plane. Here, $(u, v, w)$ are the velocities in the $(x, y, z)$ directions; $\theta$ is the potential temperature; $\theta_{0}$ is a reference potential temperature; $p$ is the pressure divided by the mean density; $g$ is the acceleration of gravity; $f$ is a (constant) Coriolis parameter; and $\left(U_{g}, V_{g}\right)$ is the horizontally uniform geostrophic flow corresponding to the mean gradient of the pressure. In the prognostic equations, $d / d t$ is defined as follows:

$$
\frac{d}{d t}=\frac{\partial}{\partial t}+u \frac{\partial}{\partial x}+v \frac{\partial}{\partial y}+w \frac{\partial}{\partial z} .
$$

The overbar - and bracket $\langle\cdot\rangle$ denote a filtering operation to extract a grid-scale variable and an average over a horizontal plane, respectively. The effects of the SGS components on the GS motion and temperature are represented in the subgrid stress terms, which are defined as follows:

$$
\begin{aligned}
\tau_{i j} & =\overline{u_{i} u_{j}}-\bar{u}_{i} \bar{u}_{j}, \\
\tau_{\theta j} & =\overline{\theta u_{j}}-\bar{\theta} \bar{u}_{j} .
\end{aligned}
$$

The subgrid stresses $\tau_{i j}$ and $\tau_{\theta j}$ must be parameterized with an SGS model in order to constitute a set of closed equations since $\overline{u_{i} u_{j}}$ and $\overline{\theta u_{j}}$ are unknown variables. We examine the following four types of SGS models, which are widely adopted in LES.

In the standard Smagorinsky model (hereafter referred to as SM), the subgrid stress is parameterized 
in the form of eddy viscosity as follows:

$$
\begin{gathered}
\tau_{i j}=-2 K_{m} \bar{S}_{i j}+\frac{1}{3} \delta_{i j} \tau_{l l}, \\
\bar{S}_{i j} \equiv \frac{1}{2}\left(\frac{\partial \bar{u}_{i}}{\partial x_{j}}+\frac{\partial \bar{u}_{j}}{\partial x_{i}}\right), \\
K_{m}=\left(C_{s} \Delta\right)^{2} \sqrt{2 \bar{S}_{i j} \bar{S}_{i j}}
\end{gathered}
$$

(Smagorinsky 1963). Here, $\Delta=\left(\Delta_{x} \Delta_{y} \Delta_{z}\right)^{1 / 3}$ represents the characteristic length of the grid spacing, and $C_{S}$ is the Smagorinsky constant. While the estimated value of the Smagorinsky constant is about 0.2 for isotropic turbulence (Lilly 1967), Deardorff (1970) reported that $C_{s}=0.1$ is optimal for turbulent channel flow. Beare et al. (2006) concluded that a value of the Smagorinsky constant of less than 0.2 is desirable for an SBL. In this study, we assume $C_{s}=0.15$, following Brown et al. (1994). The SGS temperature flux $\tau_{\theta j}$ is also parameterized in terms of eddy diffusivity as follows:

$$
\tau_{\theta j}=-K_{h} \frac{\partial \bar{\theta}}{\partial x_{j}}=-\frac{K_{m}}{\operatorname{Pr}} \frac{\partial \bar{\theta}}{\partial x_{j}},
$$

where $\operatorname{Pr}$ is the turbulent Prandtl number. There is no common formulation for $\mathrm{Pr}$ in the SM; it is often given empirically. For simplicity, $\operatorname{Pr}$ is assumed to be unity in our experiment. However, it has been indicated that the turbulent Prandtl number depends on the Richardson number (Brown et al. 1994). It should be noted that the standard Smagorinsky model does not include the production of turbulent kinetic energy due to buoyancy effects, while the buoyancy production term could be included in the Smagorinsky model (Lilly 1962).

In the dynamic Smagorinsky model (DSM) (Germano et al. 1991; Lilly 1992), the Smagorinsky constant $C_{s}$ is determined dynamically. Germano et al. (1991) introduced a test filter with a length scale of $\alpha \Delta(\alpha>1)$ and defined the subgrid stress for the test filter as follows:

$$
T_{i j} \equiv \widetilde{\overline{u_{i} u_{j}}}-\tilde{\bar{u}}_{i} \tilde{\bar{u}}_{j} \text {. }
$$

Here, the tilde $(\tilde{*})$ denotes a filtering operation with the test filter. It should be noted that the identity (called the Germano identity), indicated by

$$
\mathscr{L}_{i j}=T_{i j}-\tilde{\tau}_{i j}, \quad \mathscr{L}_{i j} \equiv \widetilde{\overline{\bar{u}}_{i} \bar{u}_{j}}-\tilde{\bar{u}}_{i} \tilde{\bar{u}}_{j},
$$

is derived from eqs. (6) and (12). When $\tau_{i j}$ and $T_{i j}$ are described by the Smagorinsky model, as in eqs. (8)-(10), we obtain the following:

$$
\begin{aligned}
& \left(C_{s} \Delta\right)^{2} \mathscr{M}_{i j}=\mathscr{L}_{i j}^{a}, \\
& \mathscr{M}_{i j} \equiv 2\left(\left|\widetilde{\bar{S} \mid \bar{S}_{i j}}-\alpha^{2}\right| \tilde{\bar{S}} \mid \tilde{\bar{S}}_{i j}\right), \\
& \mathscr{L}_{i j}^{a} \equiv \mathscr{L}_{i j}-\frac{1}{3} \mathscr{L}_{k k} \delta_{i j} .
\end{aligned}
$$

Here, scale invariance is assumed; $C_{S}$ is independent of the filter scale $\Delta$ (Germano et al. 1991). Note that because of the incompleteness of the Smagorinsky model, both sides in eq. (14) are not exactly equal. Lilly (1992) proposed to determine the most suitable value of $C_{s}$ such that the square error of eq. (14),

$$
\eta_{i j} \eta_{i j} \equiv\left(\mathscr{L}_{i j}^{a}-\left(C_{s} \Delta\right)^{2} \mathscr{M}_{i j}\right)\left(\mathscr{L}_{i j}^{a}-\left(C_{s} \Delta\right)^{2} \mathscr{M}_{i j}\right),
$$

is minimized. Following his approach, we obtain

$$
\left(C_{s} \Delta\right)^{2}=\frac{\left\langle\mathscr{L}_{k l}^{a} \mathscr{M}_{k l}\right\rangle}{\left\langle\mathscr{M}_{i j} \mathscr{M}_{i j}\right\rangle},
$$

which is the horizontal averaged value. While $C_{s}^{2}$ could also have a negative value in the above expression, we set a lower limit of $C_{s}^{2}(\mathrm{DSM}) \geq$ $-\left(C_{s}(\mathrm{SM}) / 5\right)^{2}$ to ensure numerical stability. In the present experiments, we set $\alpha=2$ so that the width of the test filter is twice that of the grid spacing. Pr is assumed to be unity as well as the SM in this study, although the dynamic model can be applied to the SGS heat flux (Moin et al. 1991).

In contrast to the SM and DSM, Deardorff (1980) introduced the prognostic equation for SGS kinetic energy (turbulent kinetic energy: TKE), and eddy viscosity and diffusivity are parameterized as a function of TKE (Deardorff model: DM). The TKE equation is written as follows:

$$
\frac{d e}{d t}=-\tau_{i j} \frac{\partial \overline{u_{i}}}{\partial x_{j}}+\frac{g}{\theta_{0}} \tau_{\theta 3}-\frac{\partial}{\partial x_{i}} \overline{u_{i}^{\prime}\left(e+p^{\prime}\right)}-\varepsilon,
$$

where $e$ and $\varepsilon$ are the TKE and its dissipation rate, respectively. The prime symbol denotes deviation from a GS variable, for example, $A^{\prime} \equiv A-\bar{A}$. The SGS terms of eq. (18) are parameterized in the form of an eddy viscosity model:

$$
\begin{aligned}
& \tau_{i j}=-2 K_{m} \bar{S}_{i j}+\frac{2}{3} \delta_{i j} e, \\
& \tau_{\theta j}=-K_{h} \frac{\partial \bar{\theta}}{\partial x_{j}}, \\
& \overline{u_{i}^{\prime}\left(e+p^{\prime}\right)}=-2 K_{m} \frac{\partial e}{\partial x_{i}},
\end{aligned}
$$


and the eddy viscosity and diffusivity coefficients are assumed to be

$$
\begin{aligned}
& K_{m}=C_{e} l e^{1 / 2}, \\
& K_{h}=\left(1+\frac{2 l}{\Delta}\right) K_{m} .
\end{aligned}
$$

Here, $C_{e}=0.10$ and

$$
l= \begin{cases}\Delta, & \frac{\partial \bar{\theta}}{\partial z} \leq 0 \\ \min \left(0.76 e^{1 / 2}\left(\frac{g}{\theta_{0}} \frac{\partial \bar{\theta}}{\partial z}\right)^{-1 / 2}, \Delta\right), & \frac{\partial \bar{\theta}}{\partial z}>0 .\end{cases}
$$

The dissipation rate of the TKE $\varepsilon$ is given as

$$
\begin{aligned}
& \varepsilon=\frac{C_{\varepsilon} e^{3 / 2}}{l}, \\
& C_{\varepsilon}=0.19+0.51 \frac{l}{\Delta},
\end{aligned}
$$

on the basis of the dimensional analysis.

The two-part model (TPM) proposed by Sullivan et al. (1994) is formulated as a modification of the Deardorff model. In this model, eddy viscosity coefficients for grid-scale eddies and horizontally uniform components are considered separately. The SGS momentum flux (19) is modified as follows:

$$
\tau_{i j}=-2 K_{m} \gamma \bar{S}_{i j}-2 K_{M}\left\langle\bar{S}_{i j}\right\rangle+\frac{2}{3} \delta_{i j} e .
$$

Here, $\gamma$ is referred to as an isotropy factor, which is defined as follows:

$$
\begin{aligned}
& \gamma \equiv \frac{S^{\prime}}{S^{\prime}+\langle S\rangle}, \\
& \langle S\rangle \equiv \sqrt{2\left\langle\bar{S}_{i j}\right\rangle\left\langle\bar{S}_{i j}\right\rangle}, \\
& S^{\prime} \equiv \sqrt{2\left\langle\left(\bar{S}_{i j}-\left\langle\bar{S}_{i j}\right\rangle\right)\left(\bar{S}_{i j}-\left\langle\bar{S}_{i j}\right\rangle\right)\right\rangle .}
\end{aligned}
$$

It should be noted that the isotropy factor $\gamma$ is unity for isotropic turbulence, and eq. (24) is identical to the DM when $\gamma=1$. The eddy viscosity coefficient for the horizontally uniform components $K_{M}$ is determined so as to match the Monin-Obukhov similarity theory (Sullivan et al. 1994).

The numerical simulation is performed with a setup following the GABLS intercomparison study (Beare et al. 2006), which is based on the idealized Arctic moderately stable boundary layer case described by Kosovic and Curry (2000). The initial potential temperature is $265 \mathrm{~K}$ up to $100 \mathrm{~m}$ with an overlying inversion strength of $0.01 \mathrm{Km}^{-1}$. The prescribed cooling rate of the surface temperature is $0.25 \mathrm{Kh}^{-1}$. The imposed uniform geostrophic flow is set to $\left(U_{g}, V_{g}\right)=(8,0) \mathrm{ms}^{-1}$, with a Coriolis parameter of $f=1.39 \times 10^{-4} \mathrm{~s}^{-1}$, which corresponds to latitude $73^{\circ} \mathrm{N}$. The domain size is set at $400 \mathrm{~m} \times 400 \mathrm{~m} \times 400 \mathrm{~m}$. Simulations are performed with isotropic grid spacings of $6.25 \mathrm{~m}$, $3.125 \mathrm{~m}$ and $2 \mathrm{~m}$, which correspond to $64^{3}, 128^{3}$, and $200^{3}$ grids, respectively.

We adopted a pseudospectral method for a horizontal plane, where the nonlinear terms are calculated in a physical space. The vertical discretization follows the Lorentz grid, in which $u, v, \theta$, and $p$ are set to integer grids and $w$ to half-integer grids. Vertical differentiation is evaluated with a second-order finite difference. Time integration is carried out for $9 \mathrm{~h}$ with the fourth-order Runge-Kutta method, and we analyze the results for the last hour.

\section{Intercomparison of first- and second-order statistics}

The vertical profile of horizontal velocity and potential temperature averaged over a horizontal plane is shown in Fig. 1. A supergeostrophic jet in the velocity and a strongly positive gradient of the potential temperature near the top of the SBL is commonly observed in all models, even in experiments with the lowest resolution. Although the differences among the models are smaller with increasing the model resolution, the height of the SBL differs by approximately $10 \mathrm{~m}$ at $2-\mathrm{m}$ resolution. The SBL depth in the SM and DSM tends to be lower than that in the DM and TPM independently of the resolution. The dependence on model resolution has different features among the SGS models. As the model resolution becomes coarser, the potential temperature gradient at the SBL top becomes remarkably weaker in the SM; by contrast, it intensifies in the DM and the TPM. The jet peak tends to be weakened in the DM at 6.25$\mathrm{m}$ resolution. The DSM result is the least sensitive to resolution. While a deeper SBL in the TPM has also been observed by Beare et al. (2006), the SBL height observed in the DSM in the present study is slightly lower than that observed by Beare et al. (2006). The inconsistency seen in the DSM could be attributed to the use of different turbulent Prandtl numbers.

Figure 2 indicates the horizontal mean of the total vertical momentum flux and buoyancy flux. As expected, the spread of these fluxes decreases with 


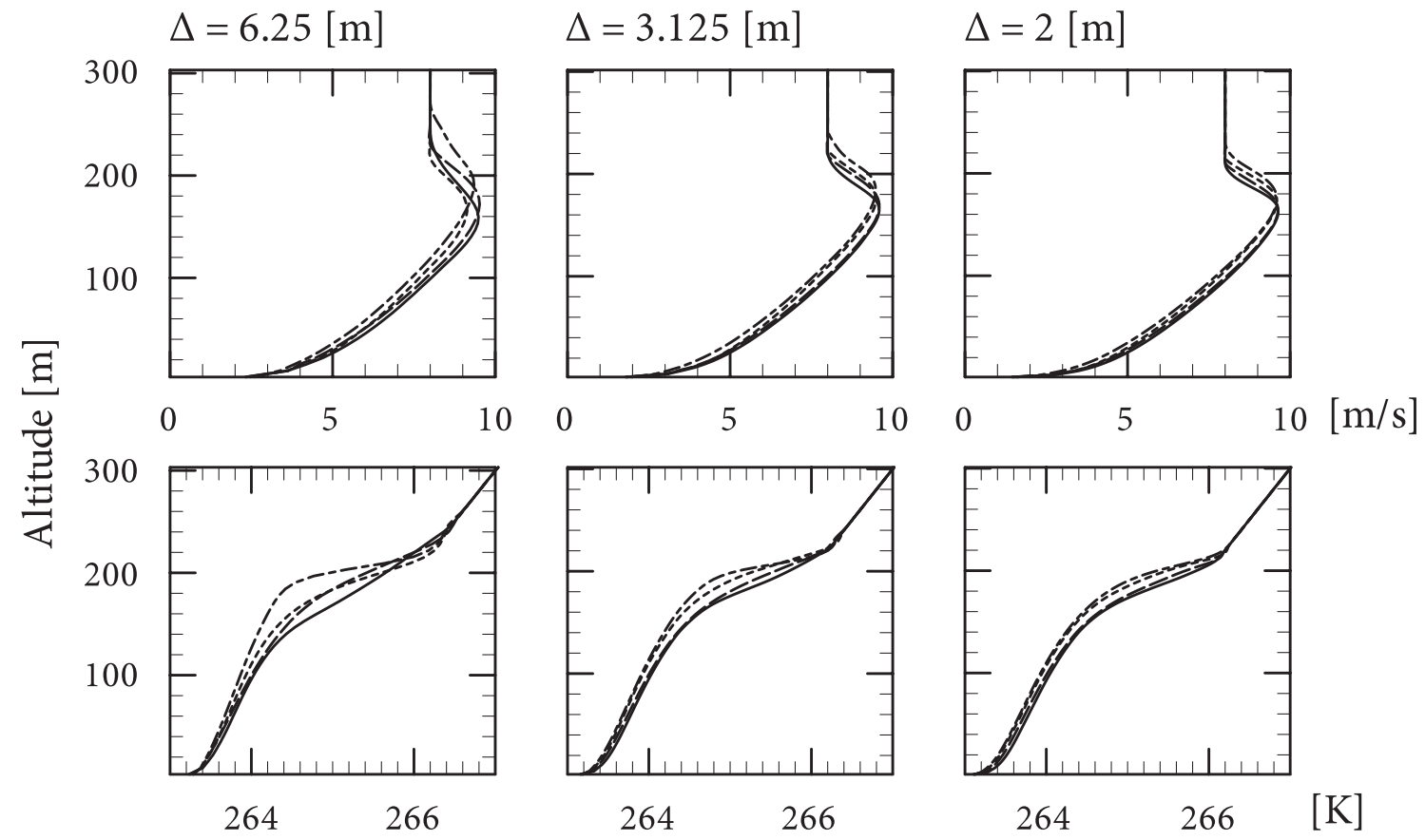

Fig. 1. Horizontal mean profiles of horizontal velocity (top) and potential temperature (bottom) at resolutions of $6.25 \mathrm{~m}$ (left), $3.125 \mathrm{~m}$ (center), and $2 \mathrm{~m}$ (right). Solid, dash, dot, and dash-dot lines indicate the results obtained from the SM, DSM, DM, and TPM, respectively.

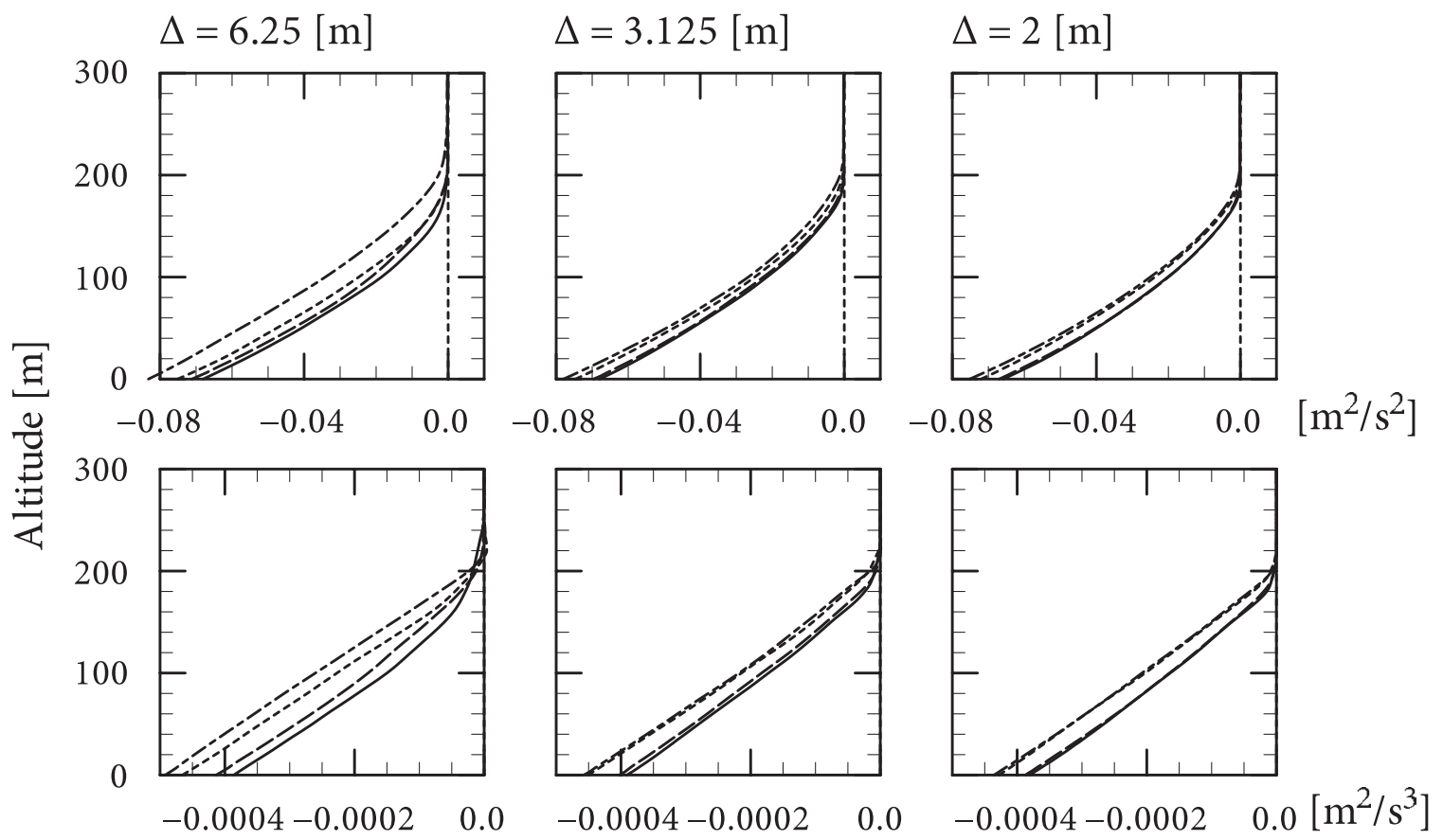

Fig. 2. Horizontal mean profiles of total momentum flux (top) and buoyancy flux (bottom). The line types are same as in Fig. 1. 


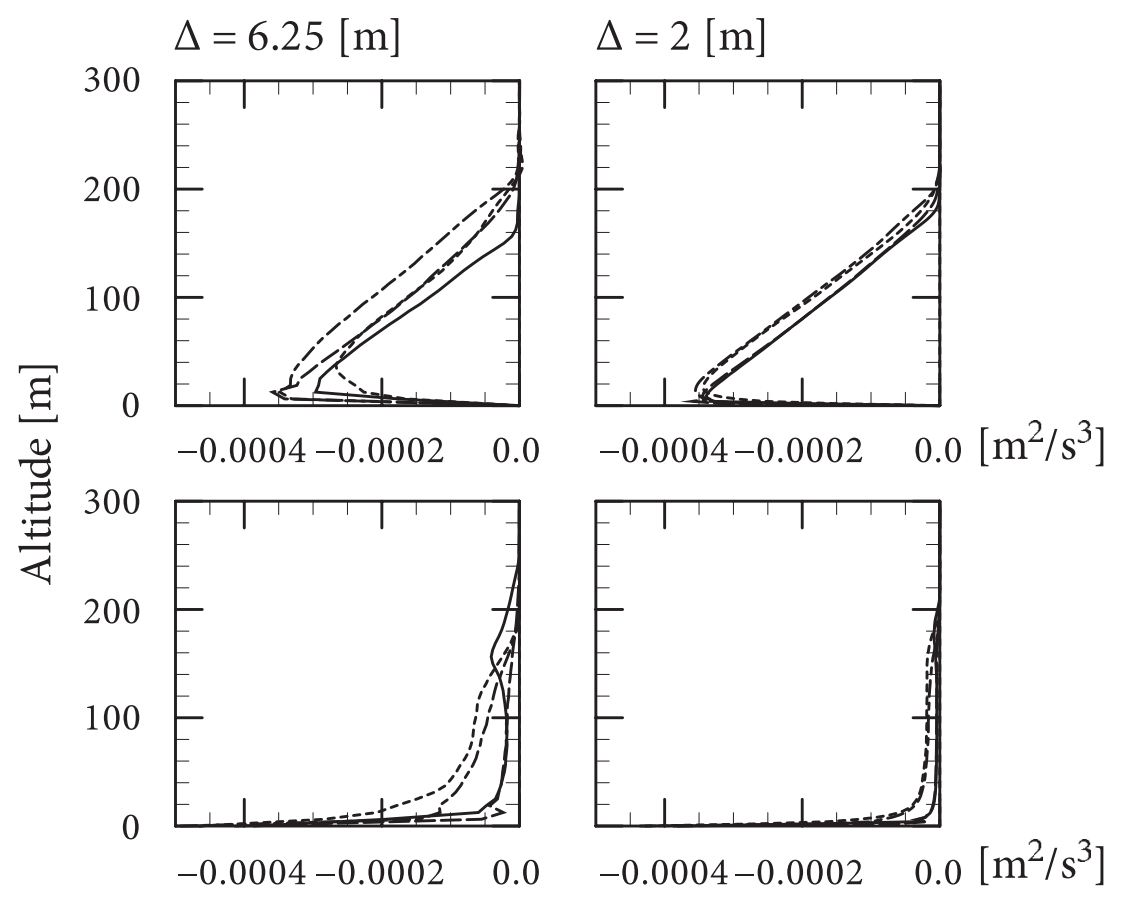

Fig. 3. Horizontal mean profiles of the GS (top) and SGS (bottom) parts of the buoyancy flux at resolutions of $6.25 \mathrm{~m}$ (left) and $2 \mathrm{~m}$ (right). The line types are same as in Fig. 1.

increasing model resolution. However, the vertical profile of these fluxes does not converge on a unique profile, even at $2-\mathrm{m}$ resolution, but instead appears to be categorized into two groups. The absolute values of the flux in the DM and TPM are apparently larger than those in the SM and DSM. This result is consistent with the mean profile seen in Fig. 1 because a larger flux value causes a deeper SBL height. The vertical gradient of the flux is insensitive to the SGS schemes and model resolution, except in the case of the SM at $6.25-\mathrm{m}$ resolution in which the vertical gradient of the buoyancy flux is weakened at the top of the SBL.

The buoyancy flux, divided into GS and SGS components, is shown in Fig. 3 to clarify the effects of SGS parameterizations. The SGS component barely contributes to the total flux, except in the vicinity of the surface where the characteristic scale of eddies is smaller because of the effect of the bottom boundary. Hence, the model resolution is sufficient to represent the most turbulent eddies in our experiments. As expected, the SGS flux decreases with increasing model resolution in all experiments. This is because the SGS flux, which is composed of spatial scales that are less than the grid size, should be reduced with increasing resolu- tion. The magnitude of the SGS flux is larger in the DM and TPM, and its tendency is remarkable at a coarse resolution $(\Delta=6.25 \mathrm{~m})$. Although the dependence of the buoyancy flux on SGS parameterizations is also reduced at finer model resolution, both the GS and the SGS components of these vertical profiles are split into two groups. Figure 3 indicates that the differences in the total flux among the models using different parameterizations could not be directly ascribed to the SGS part of the flux because they also appear in the GS part of the flux. This result is not surprising because the GS disturbance is affected by SGS components. Nevertheless, it should be noted that the GS and SGS parts of the flux tend to have a similar dependence on SGS schemes. In the model in which a larger SGS flux is observed, the GS flux also tends to be larger.

Only in the SM, the SGS flux has a negative peak at the top of the SBL, indicating that the positive potential temperature gradient at the top of the SBL is weakened by the excessive buoyancy flux. Figure 3 shows that the buoyancy flux at the SBL top is mostly caused by the SGS part. Since the SGS buoyancy flux is proportional to the potential temperature gradient in the SM where the Smagorinsky constant has an unique value in the entire do- 
main, the destruction of the structure in the SBL top could occur with a increase in grid size. This suggests that a sufficiently high resolution is necessary for the SM to reproduce an appropriate vertical profile. On the other hand, the SGS flux is suppressed in the SBL in other models, because the mixing length in the DM and TPM is shortened in the case of stable stratification or a smaller Smagorinsky constant is diagnosed in the DSM.

\section{Validation of the SGS flux based on the Germano identity}

\subsection{Objectives}

In the previous section, the horizontal mean profiles of the first variables and second-order fluxes are compared among the different SGS parameterization schemes. While this intercomparison aids in our understanding of the characteristics of each scheme, it does not elucidate how each scheme is appropriate for the reproduction of the structure of the real boundary layer. Our results indicate that the vertical profile of the first- and second-order statistics does not converge on a unique profile in models with $2-\mathrm{m}$ spatial resolution. It is uncertain whether the correct answer lies within the spread obtained from our intercomparison experiments. Even if the results of the LES models converge independently of the SGS scheme, it would be still uncertain whether the result dependably converged on the correct answer because there might be a bias that is independent of SGS modeling. A comparison to observational data is necessary to directly confirm the appropriateness of the model results. However, in contrast to a laboratory measurement, obtaining observational data to compare the LES results with sufficient precision in the atmospheric boundary layer is difficult. In such cases, validating the self-consistency of an SGS scheme using numerical data could provide useful information regarding the reliability of the SGS scheme. We propose the utilization of the Germano identity for selfconsistency testing.

The Germano identity, defined in eq. (13), represents a relation between the GS and SGS fluxes at scales between the grid and test filters. When a parameterization scheme is used to describe the SGS flux, a residual error can be included in the Germano identity due to incompleteness of the SGS parameterization. The Germano identity was originally used for the purpose of dynamically finding the Smagorinsky constant value that yields the smallest error in terms of Germano identity (Ger- mano et al. 1991; Lilly 1992). On the other hand, Meneveau and Katz (1999) proposed that it could be used to directly validate the accuracy of the SGS flux diagnosed from an LES model; they evaluated the errors in several LES models on the basis of the experimental data obtained by particle image velocimetry (PIV). Obtaining velocity or temperature field data as a snapshot is required for the self-consistency test using the Germano identity; however, satisfying this requirement appears to be difficult for observations in the atmospheric boundary layer, where an observational technique such as the PIV has not been fully established. In the present study, we examined the evaluation of residual error on the basis of the Germano identity using numerical data obtained by LES experiments rather than observational data. We used four independent datasets, which were obtained from numerical simulations with the SGS schemes examined in this study (i.e., SM, DSM, DM, and TPM) and analyzed the residual error on the basis of the Germano identity for each SGS scheme.

\subsection{Analysis procedure}

In the present analysis, the error $\eta_{i j}$ associated with the Germano identity (13) is defined as

$$
\eta_{i j}(\Delta, \alpha \Delta) \equiv\left(T_{i j}-\tilde{\tau}_{i j}\right)-\mathscr{L}_{i j} .
$$

Here, the tilde represents a filter operation with test filter width $\alpha \Delta$, as defined in Section 2. $\tau_{i j}$ and $T_{i j}$ denote the subgrid stress for the grid and test filters, respectively. The error associated with the temperature flux is defined in a similar way:

$$
\begin{aligned}
& \eta_{\theta j}(\Delta, \alpha \Delta) \equiv\left(T_{\theta j}-\tilde{\tau}_{\theta j}\right)-\mathscr{L}_{\theta j}, \\
& \mathscr{L}_{\theta j}=\widetilde{\bar{\theta} \bar{u}_{j}}-\tilde{\bar{\theta}}_{\bar{u}} .
\end{aligned}
$$

It should be noted that $T_{i j}, \tau_{i j}, T_{\theta j}$ and $\tau_{\theta j}$ are evaluated with a parameterization scheme, while the other terms on the right side are calculated directly from the reference data $\bar{u}_{i}$ and $\bar{\theta} . \eta_{i j}$, defined above, can be interpreted as the difference in the GS and SGS fluxes at scales between $\Delta$ and $\alpha \Delta$, and its positive value corresponds to the SGS flux larger than the GS flux at these scales. If the GS motion includes no error, then the value of $\eta_{i j}$ can be attributed to the parameterized SGS flux. On the other hand, errors caused by the reference data could be included due to incompleteness of the reference data obtained from the LES model when the reference data for the analysis are generated numerically. This fact would be an inevitable deficiency in 

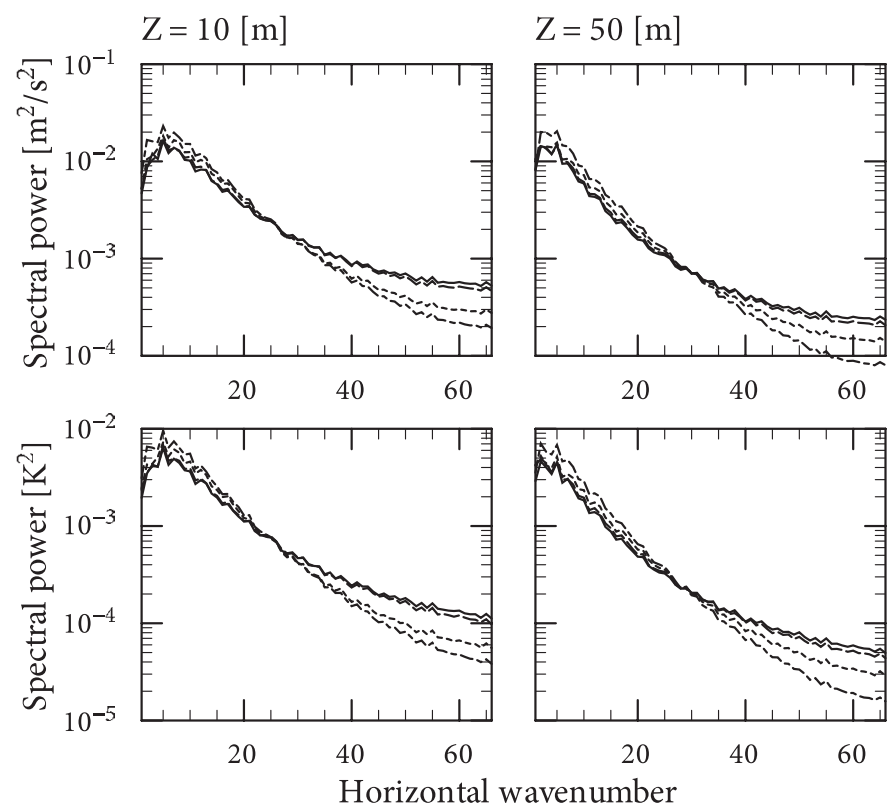

Fig. 4. Power spectrum for momentum (top) and temperature (bottom) as functions of horizontal wavenumber on a horizontal plane at $z=10 \mathrm{~m}$ (left) and $z=50 \mathrm{~m}$ (right). The results for 2-m resolution experiments are shown. The line types correspond to the adopted SGS schemes (solid: SM, dash: DSM, dot: DM, and dash-dot: TPM).

the present analysis. Nevertheless, if the value of $\eta_{i j}$ is insensitive to the reference data used for the analysis, then the robustness of the analysis could be confirmed to a certain extent.

Figure 4 displays the power spectra of momentum and temperature as a function of horizontal wavenumber on a horizontal plane at $z=10 \mathrm{~m}$ and $z=50 \mathrm{~m}$ obtained from each SGS scheme. The spread of the power spectrum among the SGS models is remarkable when it contains wavenumbers that are more than half of the truncation wavenumber. This suggests that $\eta_{i j}$, estimated as an SGS error at scales between $\Delta$ and $\alpha \Delta$, could have less reliability since the error in the GS appears to be remarkable at high wavenumbers.

To avoid difficulty in the analysis, we can evaluate the residual error by introducing a second test filter. We define a second test filter with width $\beta \Delta$ $(\beta>\alpha)$ and obtain the Germano identity, characterizing the GS and SGS fluxes at scales between $\alpha \Delta$ and $\beta \Delta$ as

$$
\begin{aligned}
& \mathscr{N}_{i j}=t_{i j}-\hat{T}_{i j}, \\
& \mathscr{N}_{i j} \equiv \widehat{\tilde{\tilde{u}}}_{i} \tilde{\bar{u}}_{j}-\hat{\tilde{\tilde{u}}}_{i} \hat{\tilde{\tilde{u}}}_{j} .
\end{aligned}
$$

Here, $t_{i j}$ and $\hat{\imath}$ are subgrid stress and filtering operations, respectively, for the second test filter. The residual error of eq. (28) is described as follows:

$$
\eta_{i j}(\alpha \Delta, \beta \Delta) \equiv\left(t_{i j}-\hat{T}_{i j}\right)-\mathscr{N}_{i j} \text {. }
$$

It should be noted that the effect of scales less than $\alpha \Delta$ is excluded in $\eta_{i j}$, as evaluated by eq. (29). We choose $\alpha=2$ and $\beta=4$, and evaluate the horizontal mean of the error $\left\langle\eta_{i j}\right\rangle$ following two types of formulae, eqs. (26) and (29).

Because the eddy viscosity coefficient is evaluated by the TKE $e$ in the DM and TPM, the TKE in the test filter space $\tilde{e}$ must be carefully diagnosed. The test filter operation to TKE $e$ does not yield an appropriate estimation of $\tilde{e}$ because $\tilde{e}$ should be regarded as a representative value of the kinetic energy at scales less than the width of the test filter $\alpha \Delta$, which should be larger than the TKE $e$. When it is assumed that the kinetic energy spectrum in the SGS satisfies the Kolmogorov $-5 / 3$ power law, the total kinetic energy included in scales less than the width of the filter is solved as follows:

$$
[e] \simeq \int_{k_{\Delta}}^{\infty} C \varepsilon^{2 / 3} k^{-5 / 3} d k=\frac{3}{2} C \varepsilon^{2 / 3} k_{\Delta}^{-2 / 3},
$$


(a)

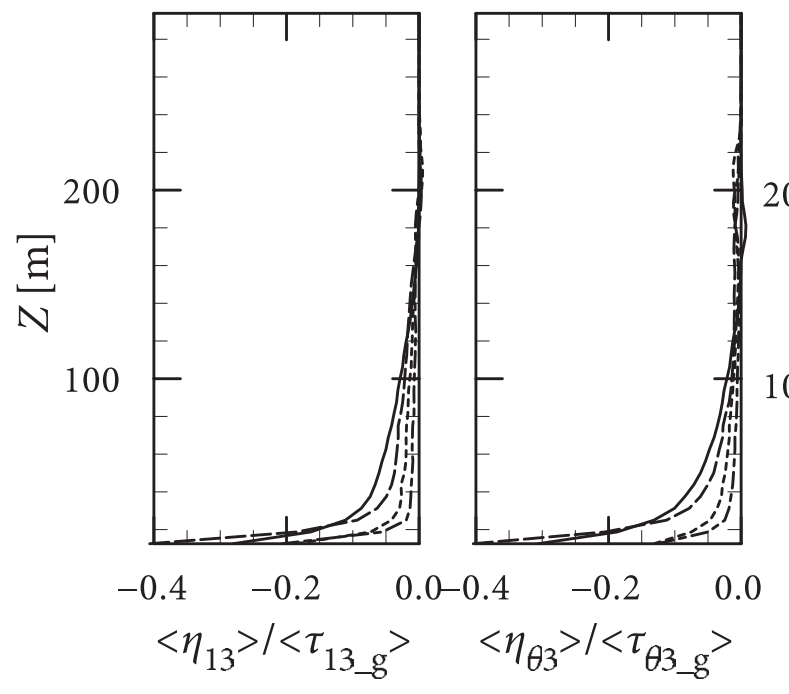

(b)

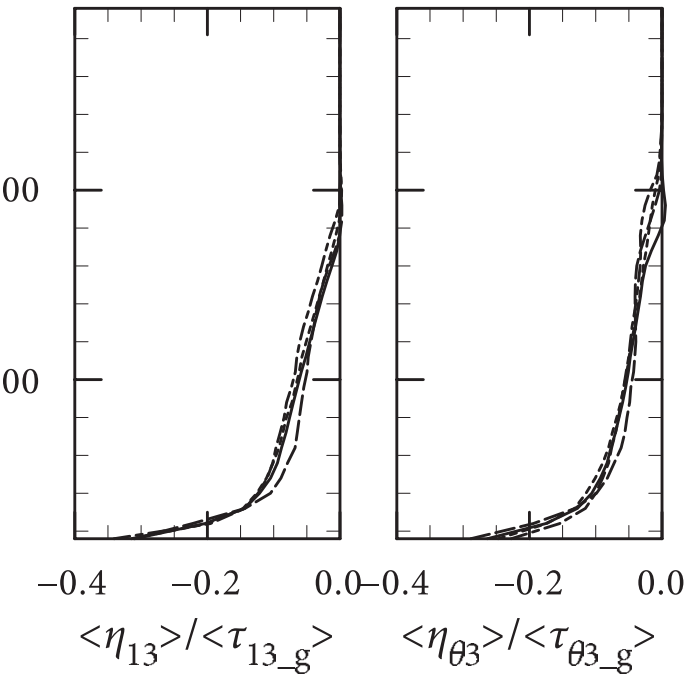

Fig. 5. The error in the Germano identity evaluated by the DSM for the vertical flux of velocity in the $x$ direction (left) and temperature (right) averaged over a horizontal plane. The plotted value is normalized by the flux at the surface. The line types indicate the SGS model used to make the reference data (solid: SM, dash: DSM, dot: DM, and dash-dot: TPM). (a) Estimation in the range of $[\Delta, 2 \Delta]$ and (b) estimation in the range of $[2 \Delta, 4 \Delta]$ are shown.

and

$$
[\tilde{e}] \simeq \int_{k_{\alpha \Delta}}^{\infty} C \varepsilon^{2 / 3} k^{-5 / 3} d k=\frac{3}{2} C \varepsilon^{2 / 3} k_{\alpha \Delta}^{-2 / 3}
$$

Here, the bracket [.] denotes an average over the domain, and $k_{\Delta}=2 \pi / \Delta$ and $k_{\alpha \Delta}=2 \pi / \alpha \Delta$ are the wavenumbers corresponding to the width of the grid and test filters, respectively. Therefore, we obtain $[\tilde{e}] /[e]=\alpha^{2 / 3}$ from eqs. (30)-(31). In the present analysis, the value of the TKE $\tilde{e}$ in the test filter space is multiplied by the factor $\alpha^{2 / 3}$ so that the above relation holds true. This assumption is consistent with the prognostic equation of the TKE (18), in which the dissipation term is parameterized on the basis of the Kolmogorov power law.

\subsection{Results}

First, we analyze the error in terms of the Germano identity on the basis of two formulae [i.e., (26) and (29)], and examine the dependence on the reference data. Figure 5 indicates the error in the Germano identity estimated for the horizontal mean of the vertical flux. In this figure, the error is estimated with the DSM, while the four reference data obtained from each SGS scheme are used for the analysis. The plotted value in Fig. 5 is nor- malized by the horizontal mean of the surface flux ${ }^{1}$. In all cases, the errors in both momentum and temperature fluxes have negative values throughout the boundary layer $(z<200 \mathrm{~m})$, independent of reference data. This suggests that the DSM tends to underestimate the SGS flux as the model resolution becomes coarser. A considerable spread among the different reference data appears in the value of the error evaluated in the range of $[\Delta, 2 \Delta]$. This can be ascribed to the error in the GS components at scales between $\Delta$ and $2 \Delta$. In particular, the absolute value of the error tends to be smaller when reference data produced by the DM and TPM are used. In these SGS models, the magnitude of the kinetic energy for high wavenumbers is smaller (Fig. 4), and the GS flux can be insufficient for high wavenumbers. Therefore, insufficient GS flux in these models can cancel out the underestimation of the SGS flux in the DSM. On the other hand, the error evaluated in the range of $[2 \Delta, 4 \Delta]$ is almost independent of the reference data. This

1 It should be noted that both the surface momentum flux $\left\langle\tau_{13_{-} g}\right\rangle$ and the temperature flux $\left\langle\tau_{\theta 3_{-} g}\right\rangle$ are negative. Hence, the plotted values in Fig. 5 are opposite in sign to $\left\langle\eta_{13}\right\rangle$ and $\left\langle\eta_{\theta 3}\right\rangle$. 

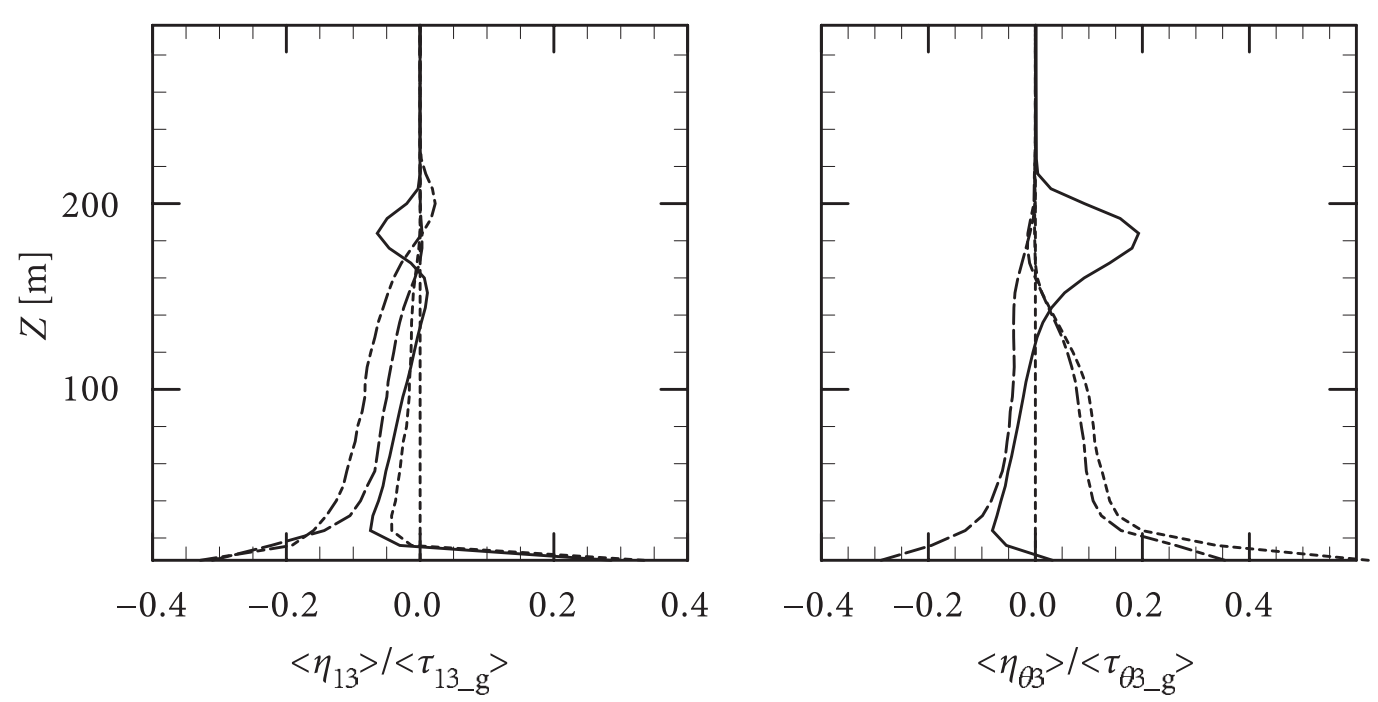

Fig. 6. As in Fig. 5, except that the results evaluated by each SGS scheme are shown. The reference data used in the analysis are reduced from $200^{3}$ to $100^{3}$ grids by the test filter. The line types indicate the SGS models used (solid: SM, dash: DSM, dot: DM, and dash-dot: TPM).

suggests that the exclusion of effects of the GS components at high wavenumbers is useful for improving the robustness of the analysis.

Figure 6 shows the error associated with the horizontal mean of the vertical flux, as estimated by the different SGS schemes. In this figure, the SGS scheme from which the reference data is obtained is same as that used for estimation of the error and the plotted value is evaluated in the range $[2 \Delta, 4 \Delta]$. The vertical profiles of $\left\langle\eta_{13}\right\rangle /\left\langle\tau_{13_{-} g}\right\rangle$ and $\left\langle\eta_{\theta 3}\right\rangle \mid\left\langle\tau_{\theta 3_{-} g}\right\rangle$ are remarkably different among the SGS schemes. In the SM, a negative peak in the momentum flux and a positive peak in the temperature flux are seen at the top of the boundary layer, while the error is relatively small in the boundary layer. In particular, the value of the positive peak in $\left\langle\eta_{\theta 3}\right\rangle$ is approximately one-fifth of the surface flux. This peak means that the SGS flux weakens excessively the gradient of the positive temperature at the top of the boundary layer for a coarser model resolution. As seen in Fig. 1, this result is consistent with the dependence of the vertical structure on model resolution. On the other hand, the DM and TPM overestimate the SGS temperature flux and underestimate the SGS momentum flux in the boundary layer at a coarser model resolution. This suggests that underestimation of the Prandtl number, defined as $\operatorname{Pr}=(1+2 l / \Delta)^{-1}$ (eq. (23)), gives rise to the excessive SGS temperature flux in these schemes.
Figures 5, 6 indicate that both the SGS momentum and the temperature fluxes, as evaluated by the DSM, are underestimated at a lower model resolution. This fact appears to be relevant to the correlation between the resolved component $\mathscr{L}_{i j}^{a}$ and the parameterized component $T_{i j}-\tilde{\tau}_{i j}$. The correlation coefficient of these anisotropic components on a horizontal plane is defined as follows:

$$
\begin{aligned}
& r(z) \\
& =\frac{\left\langle\mathscr{L}_{i j}^{a}\left(T_{i j}-\tilde{\tau}_{i j}-\mathscr{L}_{k k} \delta_{i j} / 3\right)\right\rangle}{\sqrt{\left\langle\mathscr{L}_{i j}^{a} \mathscr{L}_{i j}^{a}\right\rangle\left\langle\left(T_{i j}-\tilde{\tau}_{i j}-\mathscr{L}_{k k} \delta_{i j} / 3\right)\left(T_{i j}-\tilde{\tau}_{i j}-\mathscr{L}_{k k} \delta_{i j} / 3\right)\right\rangle}} .
\end{aligned}
$$

Using the correlation coefficient $r(z)$, we can rewrite eq. (17) as

$$
\left(C_{s} \Delta\right)^{2}=r(z) \sqrt{\frac{\left\langle\mathscr{L}_{k l}^{a} \mathscr{L}_{k l}^{a}\right\rangle}{\left\langle\mathscr{M}_{i j} \mathscr{M}_{i j}\right\rangle}}
$$

and obtain the minimized value of the square error $\left\langle\eta_{i j} \eta_{i j}\right\rangle$ :

$$
\min \left[\left\langle\eta_{i j} \eta_{i j}\right\rangle\right]=\left(1-r^{2}(z)\right)\left\langle\mathscr{L}_{i j}^{a} \mathscr{L}_{i j}^{a}\right\rangle \leq\left\langle\mathscr{L}_{i j}^{a} \mathscr{L}_{i j}^{a}\right\rangle .
$$

Equations (33) and (34) suggest that the estimated value of the Smagorinsky constant $C_{S}$ is smaller and the square error $\left\langle\eta_{i j} \eta_{i j}\right\rangle$ is larger, at smaller absolute values of the correlation coefficient. Figure 7 shows the correlation coefficient between $\mathscr{L}_{13}$ and $T_{13}-\tilde{\tau}_{13}$ (left) and that between $\mathscr{L}_{\theta 3}$ and $T_{\theta 3}-\tilde{\tau}_{\theta 3}$ 
(a)

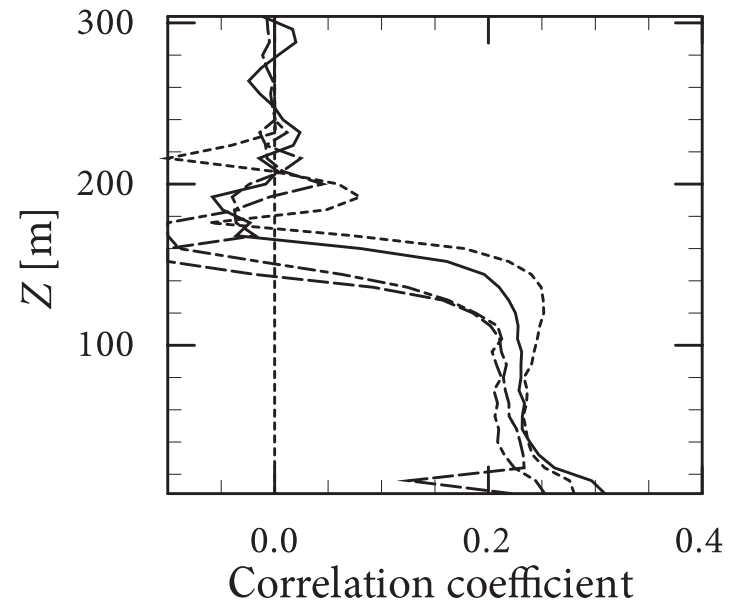

(b)

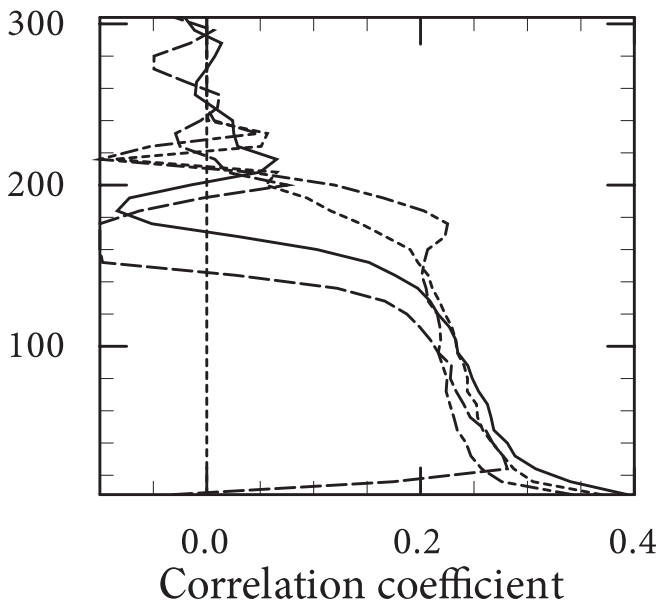

Fig. 7. Vertical profile of correlation coefficients at a horizontal plane between (a) $\mathscr{L}_{13}$ and $T_{13}-\tilde{\tau}_{13}$ and (b) $\mathscr{L}_{\theta 3}$ and $T_{13}-\tilde{\tau}_{13}$. The line types are the same as in Fig. 1.

(right), as calculated by each SGS scheme. These correlation coefficients are approximately 0.25 in the boundary layer for all schemes. This value is not sufficiently large. As a result, the dynamic procedure used in the DSM does not sufficiently contribute to a reduction of error, and the estimated SGS flux decreased. This result is consistent with that by Meneveau and Katz (1999), who analyzed a neutral stratification case. The dynamic procedure could not contribute to an improvement in the evaluation of an SGS flux unless an SGS scheme is applied such that the $T_{i j}-\tilde{\tau}_{i j}$ sufficiently correlates with $\mathscr{L}_{i j}$.

The vertical profile of the Smagorinsky constant calculated from the DSM is shown in Fig. 8. The Smagorinsky constant $C_{s}$ is not very sensitive to model resolution but becomes slightly smaller with decreasing model resolution. This result is well consistent with the scale-invariant assumption for a model constant, which is necessary to the application of the Germano identity. The value of $C_{s}$ lies in the range $0.11-0.13$ in the boundary layer, while it is smaller near the surface and at the top of the SBL. This value is smaller than $C_{s}=0.15$, which is adopted in the SM in the present study. Nevertheless, it is still uncertain whether or not a smaller value of $C_{S}$ is more appropriate for the SM because the SGS flux in the DSM appears to be underestimated, as is shown in the present analysis.

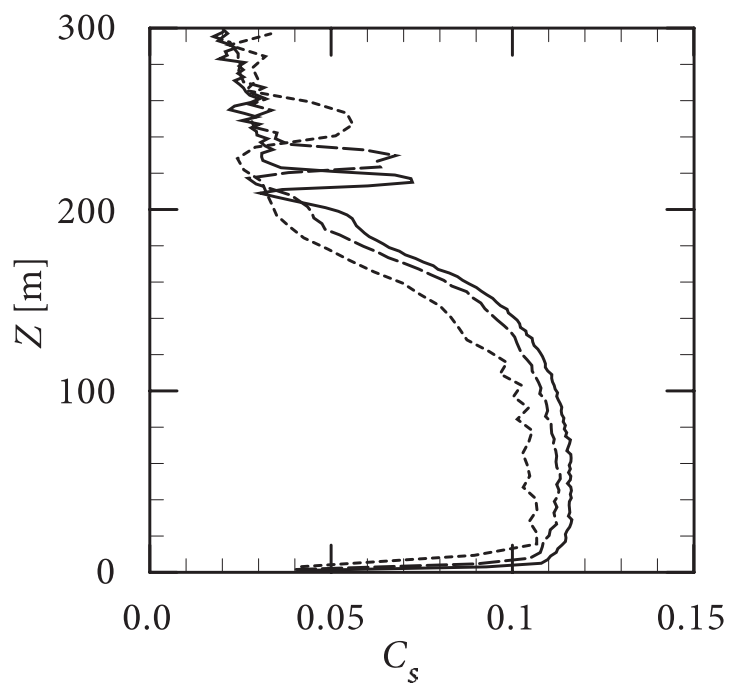

Fig. 8. Vertical profile of the Smagorinsky constant calculated from the DSM. Solid, dash, and dot lines indicate results at resolutions of $2 \mathrm{~m}, 3.125 \mathrm{~m}$, and $6.25 \mathrm{~m}$, respectively.

\section{Summary}

In the present study, numerical simulations on a moderately stable boundary layer are conducted with four SGS parameterization schemes (i.e., 
Smagorinsky, dynamic Smagorinsky, Deardorff, and two-part models), which are widely used in large-eddy simulations of the atmospheric boundary layer. In addition, dependence on the SGS scheme and model resolution is investigated. A characteristic structure in a stable boundary layer, a supergeostrophic jet in the velocity and a strongly positive gradient of the potential temperature near the top of the SBL is commonly observed in all models, even in the experiments with the lowest resolution. Although the spread of the vertical mean profile among the different SGS schemes decreases with increasing model resolution, the height of the SBL differs by about $10 \mathrm{~m}$, even for 2-m resolution. The potential temperature gradient at the SBL top is sensitive to the SGS scheme and the model resolution. While it becomes weaker in the SM, it is intensified in the DM and TPM. Results obtained from the DSM are the least sensitive to model resolution. The spread of the momentum and buoyancy fluxes also decreases with increasing model resolution. However, these fluxes tend to split into two groups rather than converge on a unique profile, even at 2-m resolution. The magnitude of the flux is apparently larger in the DM and TPM than in the SM and DSM.

Furthermore, we attempt to validate the selfconsistency of an SGS parameterization on the basis of the Germano identity to clarify the appropriateness of an SGS flux that is diagnosed by a parameterization scheme. If the effects of the GS components for higher wavenumbers are excluded from the analysis, the error estimated by the Germano identity is insensitive to the reference data, even when the reference data is generated by numerical simulations rather than observations. The SGS flux, evaluated by the SM, tends to excessively weaken the positive temperature gradient at the top of the boundary layer with decreasing model resolution. While the DM and TPM overestimate the SGS temperature flux at a coarser resolution, the DSM underestimates both the SGS momentum and the temperature fluxes throughout the entire boundary layer. This underestimation could be attributed to a low correlation between $\mathscr{L}_{i j}$ and $T_{i j}-\tilde{\tau}_{i j}$. It suggests that a dynamic procedure based on the Germano identity does not work sufficiently well unless $T_{i j}-\tilde{\tau}_{i j}$ diagnosed by a parameterization scheme highly correlates with $\mathscr{L}_{i j}$.

In the present analysis, the error estimated by the Germano identity is robust, independent of the reference data. It should be noted that the analysis proposed in the present study could be adapted to other second-order statistics (e.g., velocity variance). While the approach is expected to provide useful information about the reliability of the SGS scheme, the consistency of the results obtained in the present study is closed only in numerical models. Further study would be necessary to confirm the feasibility of the present approach.

\section{Acknowledgement}

The author would like to thank two anonymous reviewers for the constructive comments. The author is partly supported by JSPS Grant-in-Aid for Scientific Research. All the figures are drawn with the GFD-DENNOU Library (SGKS Group 1995).

\section{References}

Beare, R. J., M. K. Macvean, A. A. M. Holtslag, J. Cuxart, I. Esau, J.-C. Golaz, M. A. Jimenez, M. Khairoutdinov, B. Kosovic, D. Lewellen, T. S. Lund, J. K. Lundquist, A. McCabe, A. F. Moene, Y. Noh, S. Raasch, and P. Sullivan, 2006: An intercomparison of large-eddy simulations of the stable boundary layer. Bound.-Layer Meteorol., 118, 247-272.

Brown, A. R., S. H. Derbyshire, and P. J. Mason, 1994: Large-eddy simulation of stable atmospheric boundary layers with a revised stochastic subgrid model. Roy. Meteor. Soc., 120, 1485-1512.

Deardorff, J. W., 1970: A numerical study of threedimensional turbulent channel flow at large reynolds numbers. J. Fluid Mech., 41, 453-480.

Deardorff, J. W., 1972: Numerical investigation of neutral and unstable planetary boundary layers. $J$. Atmos. Sci., 29, 91-115.

Deardorff, J. W., 1980: Stratocumulus-capped mixed layers derived from a three-dimensional model. Bound.-Layer Meteorol., 18, 495-527.

Germano, M., U. Piomelli, P. Moin, and W. H. Cabot, 1991: A dynamic subgrid-scale eddy viscosity model. Phys. Fluids, A3, 1760-1765.

Kosovic, B., and J. A. Curry, 2000: A large eddy simulation study of a quasi-steady, stably stratified atmospheric boundary layer. J. Atmos. Sci., 57, 1052 1068.

Leonard, A., 1974: Energy cascade in large-eddy simulations of turbulent fluid flows. Advances in Geophys., A18, 237-248.

Lilly, D. K., 1962: On the numerical simulation of buoyant convection. Tellus, 14, 148-172.

Lilly, D. K., 1967: The representation of small-scale turbulence in numerical simulation experiments. Proc. IBM Sci. Comput. Symp. on Environ. Sci., IBM Form 320-1951, 195-210. 
Lilly, D. K., 1992: A proposed modification of the germano subgrid-scale closure method. Phys. Fluids, A4, 633-635.

Meneveau, C., and J. Katz, 1999: Dynamic testing of subgrid models in large eddy simulation based on the germano identity. Phys. Fluids, 11, 245-247.

Moin, P., K. Squires, W. Cabot, and S. Lee, 1991: A dynamic subgrid-scale model for compressible turbulence and scalar transport. Phys. Fluids, A3, 2746-2757.
Nieuwstadt, F. T. M., 1984: The turbulent structure of the stable, nocturnal boundary layer. J. Atmos. Sci, 41, 2202-2216.

Smagorinsky, J., 1963: General circulation experiments with the primitive equations I. the basic experiment. Mon. Wea. Rev., 91, 99-164.

Sullivan, P. P., J. C. McWilliams, and C.-H. Moeng, 1994: A subgrid-scale model for large-eddy simulation of planetary boundary-layer flows. Bound. Layer Meteorol., 71, 247-276. 